\title{
Dr. Rolf Wiegert in memoriam
}

\section{Jürgen Chlumsky • Ralf Münnich • Jürgen Schmidt}

Online publiziert: 3. Mai 2017

(C) Springer-Verlag Berlin Heidelberg 2017

Am 9. Dezember 2016 ist unser Freund und Kollege Dr. Rolf Wiegert verstorben. Für die Deutsche Statistische Gesellschaft hat er sich vor allem an der Schnittstelle zwischen amtlicher und universitärer Statistik sehr verdient gemacht.

Rolf Wiegert wurde am 18. April 1931 in Essen geboren. An der Universität Bonn begann er sein Studium, das er in Tübingen in Mathematik und Volkswirtschaftslehre abschloss. In Volkswirtschaftslehre wurde er später auch promoviert. Seine Dissertationsschrift zum Thema Schätzung bei Heteroskedastizität (Wiegert 1980), die mit dem Preis der Industrie- und Handelskammer Mittlerer Neckar ausgezeichnet wurde, demonstriert seine Fähigkeit, Theorie und Anwendung in besonderer Weise zu verbinden - ein Leitthema, das stets in seinen Arbeiten anzutreffen war.

Nach seinem Studium war er lange Zeit für das Institut für Angewandte Wirtschaftsforschung in Tübingen tätig, zunächst als Mitarbeiter und später als Berater. Hier wirkte er an der Konzeption der ersten ökonometrischen Vierteljahresmodelle für die Bundesrepublik Deutschland mit. Mit Adolf Wagner verfasste er eine Arbeit über die Zerlegung von Preisänderungen in Einkommens- und Substitutionseffekt (Wagner und Wiegert 1982).

Die Autoren danken Dr. Almut Steger und Prof. Dr. Heinz Grohmann für die Unterstützung.

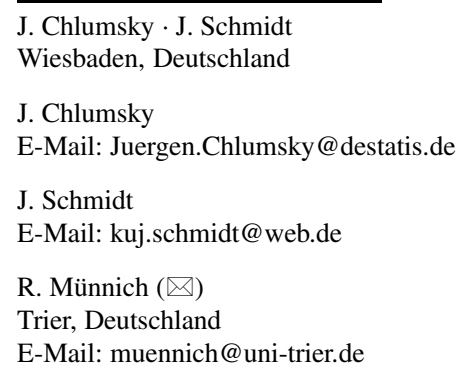


An der Universität Tübingen nahm er Lehraufgaben in Mathematik, Statistik und Datenverarbeitung wahr. Zusammen mit Professor Dr. Heinrich Strecker verfasste er eine Reihe von Schriften, die in der Erhebungsstatistik eine besondere Stellung einnehmen. Sehr bekannt sind insbesondere die Arbeiten zur Antwortvariabilität (siehe bspw. Strecker et al. 1983), die teilweise auch ins Französische übersetzt wurden, sowie zur Datenqualität (Strecker und Wiegert 1994). Unvergessen sind hier die Schweinezählungen in Belgien, die auch heute noch in Vorlesungen zur Stichprobentheorie vorgestellt werden. Ebenso unvergessen ist auch die Erstellung der zugehörigen Tabellen bis Anfang der 1990er-Jahre. Diese füllten mühelos drei A0-Poster aus und wurden mühsam von seiner Sekretärin zusammengeklebt. Auch im damaligen Allgemeinen Statistischen Archiv sind eine Reihe von Publikationen zu Stichproben und weiteren Themen aus dem Bereich der Erhebungsstatistik entstanden, wie zum Beispiel Strecker und Wiegert (1984, 1989, 1992).

Der wissenschaftliche Dialog zwischen den Statistikern der amtlichen Statistik und den Statistikern aus Universitäten und Forschungsinstituten lag Rolf Wiegert immer besonders am Herzen. Seine präzise Sprache sowie die Verbindung von Theorie und Praxis, von Ökonomie und (Erhebungs-) Statistik machten ihn zum idealen Gesprächs- und Forschungspartner für die amtliche Statistik.

So wurde der Ausschuss „Methodik Statistischer Erhebungen“ in der Deutschen Statistischen Gesellschaft, der 1986 von Professor Dr. Heinrich Strecker nach dem Vorbild der International Association of Survey Statisticians (IASS) des Internationalen Statistischen Instituts (ISI) gegründet worden war, von Rolf Wiegert in der Kooperation zwischen amtlicher und universitärer Statistik sehr erfolgreich weiterentwickelt - eine Tradition, die auch heute noch fortgeführt wird und zeigt, wie bedeutsam gerade die Kombination der beiden Richtungen ist. Rolf Wiegert leitete diesen Ausschuss acht Jahre lang von 1990 bis 1998.

Zusammen mit Jürgen Chlumsky organisierte Rolf Wiegert ein wissenschaftliches Kolloquium zur „Qualität statistischer Daten“ als gemeinsame Veranstaltung des Statistischen Bundesamts und der Deutschen Statistischen Gesellschaft. Diese Veranstaltung am 12. und 13. November 1992 war ein voller Erfolg. „Dieser positive Eindruck wurde nicht nur von vielen anderen Teilnehmern durchweg bestätigt, sondern in Gesprächen am Rande des Kolloquiums bereits in die Anregung umgesetzt, ein solches Forum des Dialogs zwischen amtlicher Statistik und ihren Nutzern, besonders aus dem Wissenschaftsbereich, als gemeinsame jährliche Veranstaltung der Deutschen Statistischen Gesellschaft und des Statistischen Bundesamtes auf Dauer zu etablieren“, schrieb der damalige Präsident des Amtes Hans-Günter Merk an Joachim Frohn (Merk 1993), zu dieser Zeit Vorsitzender der DStatG. So kam es dann auch. Regelmäßig im November trifft sich seither die Community in Wiesbaden zum Kolloquium. Kurz vor Rolf Wiegerts Tod feierte es sein 25-jähriges Bestehen mit dem Thema „Das Produktivitäts-Paradoxon - Messung, Analyse, Erklärungsansätze“.

Die ersten zehn Jahre haben sich Rolf Wiegert und Jürgen Chlumsky um Thema und Auswahl der Vortragenden gekümmert. Egal ob ,Wohlfahrtsmessung - Aufgabe der Statistik im gesellschaftlichen Wandel“ (1995), „Statistische Informationen zum Arbeitsmarkt - Konzepte und Kritik, Anwendung und Auslegung“ (1996) oder aber „Familien und Haushalte in Deutschland - Statistische Grundlagen, wissenschaftli- 
che Erkenntnisse“ (2000) thematisiert wurden, stets ging es um den Dialog zwischen Wissenschaft, amtlicher Statistik und den Nutzern amtlicher Statistiken aus Politik und Wirtschaft. Zum 50-jährigen Bestehen der Bundesrepublik Deutschland wählten Wiegert und Chlumsky als Thema „Amtliche Statistik - Ein konstitutives Element des demokratischen Staates“.

In schöner Erinnerung bleiben den Beteiligten die Planungstreffen im Mössinger Garten von Rolf Wiegert, stets auch ergänzt durch einen Waldspaziergang mit seinem treuen vierbeinigen Begleiter Hektor.

Die Krönung des Schaffens eines Survey-Statistikers ist ohne Zweifel die Mitarbeit an einem Zensus. So war Rolf Wiegert bei den Beratungen zum Zensustest 2001 beteiligt, einem Quantensprung in der Methodik, dem Übergang von der klassischen Volkszählung zum registergestützten Zensus. Unter seiner Leitung wurde eine ganztägige und sehr erfolgreiche Sonderveranstaltung zu diesem Thema im Rahmen der Statistischen Woche 1998 durchgeführt, welche als Sonderband erschien (Grohmann et al. 1999). Wie wir heute wissen, wurde 2011, nach der Testerhebung im Jahr 2001, der erste registergestützte Zensus durchgeführt.

Nach seinem Eintritt in den Ruhestand unterstützte Rolf Wiegert weitere Forschungsprojekte. So war er Berater des Koordinators des EU-Projektes DACSEIS zur Datenqualität in komplexen Erhebungen (Münnich und Wiegert 2001). Sein Enthusiasmus zeigte sich auch darin, dass er, um beim Treffen in Helsinki anwesend sein zu können, erst- und einzigmalig ein Flugzeug bestieg. Auch das Nachfolgeprojekt Knowledge Economy Indicators (KEI) unterstützte er noch aktiv. Seine Ruhe und Übersicht auf der einen Seite sowie andererseits das hervorragende Gespür für die Verbindung von Theorie und praktischen Notwendigkeiten gaben stets ein Vorbild für seine Kolleginnen und Kollegen.

Rolf, nicht nur wir vermissen Dich, wir werden Deine Vision, statistische Theorie mit der Anwendung in der Amtlichen Statistik zu verbinden, weiterverfolgen.

\section{Literatur}

Grohmann H, Sahner H, Wiegert R (1999) Volkszählung 2001 - Von der traditionellen Volkszählung zum Registerzensus. All Stat Arch, Sonderheft 33

Merk HG (1993) Brief an Joachim Frohn, 15. Januar.

Münnich R, Wiegert R (2001) The DACSEIS project / IST-2000-26057 DACEIS. DACSEIS Research Paper Series 1 .

Strecker H, Wiegert R, Peeters J, Kafka K (1983) Messung der Antwortvariabilität auf Grund von Erhebungsmodellen mit Wiederholungszählungen. Angewandte Statistik und Ökonometrie. Vandenhoeck \& Ruprecht, Göttingen

Strecker H, Wiegert R (1984) Der mean square error und die Antwortvarianz bei Erhebungen: Darstellung und Schätzung. All Stat Arch 68:199-222

Strecker H, Wiegert R (1989) Antwortvariabilität und Adjustierung. All Stat Arch 73:255-285

Strecker H, Wiegert R (1992) Geschichtete Stichproben und Messung der Antwortvariabilität. All Stat Arch 76:240-267

Strecker H, Wiegert R (1994) Stichproben, Erhebungsfehler, Datenqualität. Reihe Statistik und Ökonometrie. Vandenhoeck \& Ruprecht, Göttingen

Wagner A, Wiegert R (1982) Probleme der Adäquation bei Einkommenseffekt und Substitutionseffekt von Preiseänderungen. Jahrb Natl Okon Stat 197(1):21-41

Wiegert R (1980) Schätzung bei Heteroskedatizität. Tübinger wirtschaftswissenschaftliche Abhandlungen 28. Mohr, Tübingen 